\title{
O Conceito de Mecanismos de Defesa e a sua Avaliação: Alguns Contributos
}

\section{The Definition of Defense Mechanisms and their Assessment: Some Contributions}

\author{
Rui C. Campos ${ }^{1}$
}

\begin{abstract}
Resumo
Neste artigo apresentam-se diferentes perspectivas sobre o que são e qual a função dos mecanismos de defesa, nomeadamente as perspectiva de A. Freud, Vaillant, Cramer e de Ihilevich e Gleser. De seguida, descrevem-se alguns instrumentos de avaliação de mecanismos de defesa que decorrem ou operacionalizam as perspectivas referidas. Dá-se um enfoque especial ao Defense Mecahnisms Inventory (Ihilevich \& Gleser, 1986) que operacionaliza a perspectiva dos autores e que permite obter resultados para cinco escalas: Turning Against Object, Projection, Principalization, Turning Against Self e Reversal. Contrariamente a uma abordagem maturacional/evolutiva, esta perspectiva assume que os diferentes tipos de mecanismos de defesa podem ser igualmente adaptativos, dependendo da sua flexibilidade e do grau em que preservam o teste da realidade. Descreve-se ainda o Children Aperception Test (CAT; Bellak \& Bellak, 1949, 1950) enquanto instrumento que permite a avaliação dos mecanismos de defesa em crianças.
\end{abstract}

Palavras-chave: mecanismos de defesa, avaliação, Inventário de Mecanismos de Defesa, Children Aperception Test

\begin{abstract}
In this article we present different perspectives on what are and what is the function of defense mechanisms, namely the perspectives of A. Freud, Vaillant, Cramer and Ihilevich and Gleser. Then we describes some instruments for assessing defense mechanisms that follow or operationalize the aforementioned perspectives. In this paper there is a special focus on the Defense Mecahnisms Inventory (Ihilevich \& Gleser, 1986) that operationalizes the author's perspective and allows to obtain results for five scales: Turning Against Object, Projection, Principalization, Turning Against Self and Reversal. Differently to a maturational, evolutionary approach, this perspective assumes that the different types of defense mechanisms may be equally adaptive, depending on their degree of flexibility and on the degree that they preserve reality testing. the Children Aperception Test (CAT; Bellak \& Bellak, 1949, 1950) is also presented as an instrument that allows the assessment of defense mechanisms in children.
\end{abstract}

Keywords: defense mechanisms, assessment, Defense Mechanisms Inventory, Children Aperception Test

Nota de autor: O texto desde artigo é, com algumas alterações, similar a parte do relatório de unidade curricular defendido em provas agregação em psicologia clínica do autor na Universidade de Lisboa.

\footnotetext{
${ }^{1}$ Departamento de Psicologia, Escola de Ciências Sociais e Centro de Investigação em Educação e Psicologia (CIEP), Universidade de Évora, Apartado 94, 7002-554 Évora, Portugal. E-mail: rcampos@ uevora.pt
} 


\section{Introdução}

Neste artigo começaremos por tecer algumas considerações teóricas relativas à definição de mecanismo de defesa, bem como a diferentes perspectivas sobre o constructo. Passaremos depois para uma descrição de procedimentos de avaliação, apresentando instrumentos de avaliação de mecanismos de defesa, muito em particular dois instrumentos de natureza diferente: o Defense Mechanisms Inventory (Ihilevich \& Gleser, 1986), um método de resposta fechada ou de escolha forçada (veja-se Macmillan e Creelman, 2005) e, o Children Aperception Test (CAT), um método projectivo. O objectivo é, por um lado, centramonos em instrumentos que apresentem uma fundamentação teórica sólida e, por outro, que possam ser aplicados a pessoas de diferentes idades. O DMI tem uma versão para adolescentes e outra parta adultos e o CAT pode ser aplicado a crianças.

\section{Diferentes perspectivas sobre os mecanismos de defesa}

$\mathrm{O}$ conceito de mecanismo de defesa em Psicologia surge pela primeira vez em 1894 no trabalho denominado As Psiconeuroses de Defesa onde Freud descrevia a luta do Ego contra afectos dolorosos e insuportáveis para o indivíduo. Posteriormente, Freud formula o conceito de recalcamento que ocorreria devido à angústia e que resultaria e se manifestaria simbolicamente em sonhos, actos falhados e sintomas neuróticos. Mais tarde ainda, Freud (1926) viria a substituir o conceito de repressão pelo conceito de defesa (Laplanche \& Pontalis, 1988). Freud (1926) propôs diversos mecanismos de defesa, como a regressão, o recalcamento, a formação reactiva, o isolamento, a introjecção, a projecção e a anulação, sendo que mais tarde Anna Freud (1936) introduziu outros mecanismos de defesa, como a sublimação, o deslocamento, a negação e a identificação com o agressor (Mullen, Blanco, Vaughan, Vaughan, \& Roose 1999). Freud definiu os mecanismos de defesa como operações mentais ou métodos utilizados pelo Ego para se proteger contra a ansiedade (Hovanesian, Isakov, \& Cervellione, 2009). Seriam processos inconscientes que protegeriam a mente de sentimentos e pensamentos difíceis de lidar ou de impulsos carregados de desejo e que o Ego consideraria perigosos ou que entravam em conflito com as exigências do Super-ego (Vaillant, 1992).

Ao longo do século passado, a investigação sobre os mecanismos de defesa foi conduzida no âmbito de vários quadros teóricos, especialmente nas abordagens psicanalíticas. Os trabalhos de A. Freud (1946) constituem um marco fundamental na teoria e investigação sobre o tema (Cramer, 2000). A. Freud descreve as defesas patológicas como um desequilíbrio em certos processos que podem reflectir a presença de mecanismos rígidos que perturbam o desenvolvimento da personalidade. Esta autora segue uma abordagem ontogénica em relação ao desenvolvimento dos mecanismos de defesa e considera que o processo de maturação pessoal inclui uma maturação dos mecanismos de defesa (Schauenburg, Willenborg, Sammet, \& Ehrenthal, 2007). A. Freud (1946, 1965) destaca também que todos os mecanismos de defesa podem constituir modos de adaptação saudáveis, desde que sejam utilizados de forma flexível e moderada. Em sua opinião, os mecanismos de defesa ajudam os indivíduos a lidar com as exigências e desafios presentes na realidade. Desenvolver-se-iam e seriam utilizados pelo Ego como forma de lidar com os conflitos provocados pelo Id na sua relação com o Ego e com o Super-ego.

Outros autores, como Haan (1963), consideram todos os mecanismos de defesa, em todas as circunstâncias, como respostas desadaptativas do sujeito, pelo que estariam associadas ao funcionamento patológico da personalidade.

Uma terceira posição considera algumas defesas particulares como primitivas, imaturas ou patológicas, enquanto outras, como maduras e adaptativas. Trata-se de uma posição defendida, por exemplo, por Vaillant $(1971,1977)$ e Cramer (1991). Os trabalhos destes dois autores constituem contributos importantes e mais recentes para o estudo dos mecanismos de defesa, apresentando uma nova perspectiva acerca da sua função. Vaillant (1971) apresentou um modelo hierárquico dos mecanismos de defesa com quatro níveis. Agrupou dezoito defesas, com base no grau de maturidade e importância do ponto de vista psicopatológico. No primeiro nível incluem- 
se os mecanismos psicóticos (negação, distorção, projecção delirante); no segundo nível situam-se os mecanismos imaturos (fantasia, projecção, hipocondria, comportamento passivo-agressivo e acting-out); no terceiro nível estão presentes os mecanismos neuróticos (intelectualização, recalcamento, formação reactiva, deslocamento e dissociação); por último, situam-se no quarto nível os mecanismos maduros/evoluídos (sublimação, altruísmo, supressão, antecipação e humor).

De acordo com Vaillant (1971) a função dos mecanismos de defesa é a de manter os afectos dentro de limites suportáveis, redireccionar impulsos, promover alterações no auto-conceito e gerir conflitos não resolvidos com os outros. De acordo com este autor, o desenvolvimento é acompanhado pela evolução dos processos defensivos que vão desde o primeiro até ao quarto nível de maturidade (Vaillant, 1977). Cramer (1991) propôs um modelo desenvolvimental baseado na ideia que diferentes defesas emergem em diferentes etapas do desenvolvimento. De acordo com este modelo, a utilização da negação é predominante durante os anos pré-escolares, mas perde importância a meio da infância, onde é utilizada maioritariamente a projecção, sendo que esta se vai tornar predominante durante o período da adolescência. Por sua vez, a identificação, uma defesa com uma complexidade considerável, desenvolve-se lentamente na primeira infância, atravessa a adolescência, tornando-se a defesa predominante na idade adulta (Cramer, 2002).

Para Cramer (1998, 2000, 2006), os mecanismos de defesa têm uma dupla função: por um lado, proteger o indivíduo de experienciar uma ansiedade excessiva e, por outro, proteger a integridade do Self. Segundo esta autora, existem também estratégias conscientes que o indivíduo utiliza ao vivenciar situações de stress, estratégias que lhe permitem encontrar soluções realistas de forma a reduzir a ansiedade - estratégias de coping. Considera que existem duas diferenças fundamentais entre mecanismos de defesa e estratégias de coping. A primeira diferença reside no facto das estratégias de coping envolverem esforços conscientes, enquanto os mecanismos de defesa ocorrerem de forma inconsciente. A segunda diferença consiste no facto das estratégias de coping serem utilizadas com a intenção de modificar a situação/resolver o problema, enquanto os mecanismos de defesa pretendem modificar um estado psicológico interno desagradável, não tendo, contudo, efeito na realidade externa e podendo resultar numa percepção distorcida dessa realidade (Cramer, 1998, 2000).

A perspectiva de Ihilevich e Gleser (1986) afasta-se das posições de Cramer e de Vaillant. Ihilevich e Gleser consideram que, a classificação de Vaillant, por exemplo, não está, de acordo ou é incompatível com muitos dos relatórios clínicos e estudos empíricos que mostram que as pessoas, independentemente do nível de funcionamento, utilizam a maioria das defesas, mas com graus diferentes de manutenção do teste de realidade. $\mathrm{O}$ que é importante, de acordo com Ihilevich e Gleser, não é o tipo de defesas utilizadas, mas a rigidez com que são usadas e o grau de distorção da realidade que provocam. De acordo com estes autores, os mecanismos de defesa servem para distorcer ou manusear a realidade quando os recursos pessoais, competências e motivações são insuficientes para resolver conflitos internos ou ameaças externas ao bem-estar do sujeito. Ainda segundo Ihilevich e Gleser, os mecanismos defensivos são atribuídos a processos inconscientes que são activados automaticamente quando são percebidas ameaças dolorosas para serem enfrentadas de outra forma.

Ao contrário de Vaillant e Cramer, Ihilevich e Gleser consideram todos os tipos de defesa adaptativos se utilizados de forma flexível. As defesas são patológicas quando resultam em respostas rígidas, excessivas ou inapropriadas. Assim, o que define a patologia, não é tanto o tipo de defesas utilizadas, mas sim a rigidez e a frequência com que são utilizadas e, o grau de distorção da realidade que provocam (Ihilevich \& Gleser, 1986). Ihilevich e Gleser não utilizaram um critério evolutivo ou adaptativo para classificar as defesas e não consideram viável a avaliação das defesas de forma isolada. Ao contrário, agruparam as respostas defensivas ao conflito em torno de cinco estilos distintos, cada um caracterizado por um conjunto único de operações mentais, respostas emocionais e reacções comportamentais: Turning Against Sel (O voltar da agressividade contra o próprio), Turning Against Object (O voltar da agressividade 
contra o outro), Reversal (Inversão), Principalization (Neutralização) e Projection (Projecção). Note-se ainda que as cinco categorias de Ihilevich e Gleser abrangem a maioria das defesas descritas na literatura psicanalítica.

Parece-nos que esta perspectiva classificatória é rica e integradora e, permitirá mesmo, obviar um problema que se pode colocar na prática da avaliação psicológica. A questão que se pode colocar é: será possível avaliar de forma rigorosa e válida as diversas defesas isoladamente? Este é um problema que se pode colocar numa avaliação mais formal deste constructo, mas também, e sobretudo, numa avaliação informal, em entrevista. Será que é possível dizer, por exemplo, que uma dada pessoa utiliza a intelectualização e a formação reactiva num dado contexto e noutro, a negação e a racionalização? Por ouro lado, se o sujeito nos dá um exemplo de uma situação real conflitual em que é evidente que utilizou a identificação projectiva e a negação, por exemplo, isso não implica que não tenha utilizado simultaneamente outras defesas, e também, o que é igualmente importante, que utilize de forma mais sistemática essas defesas noutras situações. Pode tratar-se de reacções defensivas caracteriológicas ou, diferentemente, de reacções defensivas situacionais, para utilizar a terminologia de McWillimas (2004), aquelas que avaliamos a partir de uma ou duas situações concretas.

Diversos estudos (e.g., Azibo, 2007; Perry \& Kardos, 1995; Vaillant, 1992) concluem que a saúde mental e o bem-estar estão associados a estilos defensivos mais evoluídos, enquanto um funcionamento menos adaptativo está associado a estilos defensivos mais imaturos.

Os mecanismos de defesa mostraram utilidade em diversas áreas de investigação em Psicopatologia, por exemplo, a organização borderline da personalidade (Chabrol \& Leichsenring, 2006; Perry \& Cooper; 1986) as perturbações da personalidade (e.g., Berman \& McCann, 1995; Bowins, 2010; Cramer, 1999a; Vaillant \& Drake, 1985), as perturbações da ansiedade (e.g., Chávez-León, Muñoz, \& Uribe, 2006) e a depressão (e.g., Mullen et al., 1999; Hovanesian et al., 2009). Azibo (2007), por exemplo, encontrou uma relação entre mecanismos de defesa menos evoluídos e diversos tipos de sintomas psicopatológicos, como os depressivos e os de ansiedade. Kwon e Lemon (2000) referem que um estilo de defesa imaturo se associa à propensão para a depressão (veja-se também Campos, Besser e Blatt, 2011).

Os mecanismos de defesa também foram estudados no âmbito da investigação sobre mudança terapêutica (e.g., Bowins, 2006, Cramer, 1999b, 2005, Cramer \& Blat, 1990, 1993; Ihilevich \& Gleser, 1991, 1995). Por exemplo, Cramer e Blatt (1990), numa avaliação realizada no final da psicoterapia com jovens adultos com patologia grave, com uma duração média de quinze meses (e utilizando o TAT para avaliação dos mecanismos de defesa), verificaram, por comparação com a avaliação prévia à intervenção, um declínio no uso de defesas não adaptativas, redução que se associou a um declínio na sintomatologia psiquiátrica.

\section{Instrumentos para a avaliação dos mecanismos de defesa}

Um grande número de instrumentos tem sido proposto com o objectivo de avaliar os mecanismos de defesa. Para além do Defense Mechanisms Inventory (Ihilevich \& Gleser, 1986), que operacionaliza o modelo dos autores, diversos outros instrumentos têm sido utilizados com o objectivo referido, como por exemplo, algumas escalas do Rorschach (Cooper, Perry, \& Arrow, 1988), do TAT (Cramer, 1991) e do CAT (Boekholt, 2000; Cramer, 1991), a Defense Mechanisms Rating Scale (DMRS; Perry \& Cooper, 1986), o Defense Style Questionnaire (DSQ; Bond, Gardner, Christian, \& Sigal, 1983), o Defense Mechanisms Test (DMT; Kragh, 1955) e o Defense Mechanisms Questionnaire (DMQ; Kreitler \& Kreitler, 1972). Um instrumento interessante para avaliação de um tipo específico de mecanismo de defesa, a clivagem, de acordo com a concepção de Kernberg (1985) e, que foi objecto de estudo relativamente recente em Portugal (Silva, 2014), é o Spliting Index (Gould, Prentice, \& Ainslie, 1996).

É de notar que, com o surgimento de diversos instrumentos de avaliação dos mecanismos de defesa, uma nova era surgiu, sendo que a investigação sobre este constructo "alastrou-se" a muitas áreas da Psicologia, quando estava 
inicialmente circunscrita aos trabalhos dos psicanalistas (Justo, Melo, \& Ferreira, 2010).

De entre os vários instrumentos, pela simplicidade de aplicação e cotação e, pela quantidade de investigação realizada, pode destacar-se o Defense Style Questionnaire (DSQ). A construção do DSQ baseia-se no pressuposto de que as representações conscientes das defesas inconscientes e automáticas podem ser identificadas pelo auto-relato dos sujeitos (Flett, Besser, \& Hewitt, 2005). O DSQ foi desenvolvido com o intuito de operacionalizar processos intrapsíquicos, permitindo que os mecanismos de defesa fossem estudados objectivamente (Segal, Coolidge, \& Mizuno, 2007).

Note-se que existem várias versões deste instrumento com um número variável de itens, nomeadamente, $88,36,40$ e 60 itens, devido a tentativas consecutivas de melhorar as suas qualidades psicométricas, consideradas inicialmente fracas (Thygesen, Drapeau, Trijsburg, Lecours, \& Roten 2008). Este questionário foi originalmente desenvolvido por Bond et al. (1983), para operacionalizar e avaliar os derivados conscientes das defesas. Era constituído por 88 itens e avaliava quatro estilos defensivos: adaptativo, de auto-sacrifício, de distorção das imagens e de padrões de acção desadaptativos. Uma versão mais recente é o DSQ-60 (Trijsburg, Bond, \& Drapeau, 2003), construído de modo congruente com a perspectiva do DSM-IV (Thygesen et al., 2008). Diversas análises factoriais conduziram a diferentes soluções factoriais e, por conseguinte, a diferentes propostas de estilos defensivos, em função da saturação dos itens individuais (defesas específicas) nesses factores. Nesta versão, as diferentes defesas saturam em três factores/estilos defensivos, adaptativo, de regulação do afecto e de distorção da realidade. O DSQ-40 (Andrews, Singh, \& Bond, 1993) considera também três estilos: imaturo, neurótico e maduro (veja-se Ruuttu et. al., 2006). O DSQ-40 foi adaptado para a população portuguesa por Oliveira (2012).

\section{O Defense Mechanisms Inventory e o Defense Mechanisms Inventory-Youth}

O Defense Mechanisms Inventory (DMI; Gleser \& Ihilevich, 1969) é um dos instrumentos mais utilizados para avaliação dos mecanismos de defesa (e.g., Drageset \& Lindstrom, 2003, La Gruta et al., 2006) e tem como pressuposto que a principal função das defesas é a resolução dos conflitos entre o que é percebido pelo indivíduo e os seus valores internos. O conflito é resolvido através de um processo onde o Ego ataca, distorce ou se torna selectivo de forma inconsciente em relação a determinados aspectos do mundo interno ou externo (Gleser \& Ihilevich, 1969).

Existem duas versões do instrumento, uma para sujeitos do sexo masculino e outra para sujeitos do sexo feminino e em ambas são apresentadas 10 pequenas vinhetas descrevendo situações conflituais da vida quotidiana. Pede-se ao sujeito para escolher, relativamente a cada vinheta, em blocos de cinco opções (uma opção para cada um dos cinco tipos de mecanismos de defesa operacionalizados pelo inventário), as opções que estão mais e menos perto de sua opinião. Os quatro blocos de opções para cada vinheta estão relacionados com: o que a pessoa faria em termos de comportamento real, na fantasia, o que pensaria e o que sentiria.

Um exemplo de uma vinheta ou situação é: "Você está à espera do autocarro na esquina da rua. As ruas ficaram molhadas e lamacentas depois das chuvas da noite passada. Um carro, em alta velocidade, passa junto ao passeio e dá-lhe um duche de lama".

E o primeiro bloco de opções de resposta é: "Qual seria a sua reacção (comportamento real)?"

"Tomaria nota da matrícula, para poder identificar esse condutor perigoso.

Censurava-me por não ter, ao menos, vestido uma gabardine.

Sorrindo, limparia os pingos com um lenço.

Resignado, lembrava-me que, no fim de contas, estas coisas acontecem.

Gritava insultos ao condutor."

Outro exemplo é:

"É quase de noite. Você conduz o seu carro na cidade e pára num sinal luminoso. Quando a luz muda para o verde, você repara que os peões não obedecem ao sinal e atravessam-se no seu caminho. Tal como a lei recomenda, você avança cautelosamente antes que o sinal vermelho apareça de novo. No momento de se safar, um polícia de trânsito manda-o encostar e acusa-o de ter ofendido o direito de circulação dos peões. Você explica que procedeu da única forma 
possível. No entanto, o polícia passa-lhe uma multa".

"Se reagisse sem pensar (na fantasia) o que é que fazia?"

"Dizia ao polícia que ele não tem o direito de usar a sua posição para me espezinhar.

Censurava-me por não ter esperado pela luz verde seguinte.

Agradecia ao polícia por me ter salvo de um possível acidente.

Defendia os meus direitos por uma questão de princípio.

Batia-lhe com a porta na cara e continuava a conduzir.

Note-se que o sistema de cotação proposto pelos autores, é de cariz ipsativo, em que em cada bloco, a escolha de uma das opções de resposta condiciona a escolha das outras o que, por si só, pode constituir uma limitação. Na literatura, este tipo de escala de resposta tem sido sujeito a críticas (veja-se Moreira, 2004). Devido a estas dificuldades, D'Angelo, San Martini e Tomasello (2000) propuseram uma escala de Likert para a reposta às cinco opções dentro de cada bloco. Note-se, no entanto, que a versão portuguesa mantém o mesmo formato de resposta da versão original.

Não deixando o DMI de ser um instrumento de auto-relato, difere em diversos aspectos, para além do já referido formato dos "tens", relativamente aos questionários que avaliam mecanismos de defesa, como o Defense Style Questionnaire (Bond et al., 1983). Pensamos que o DMI apresenta diversas vantagens: por um lado, a formulação teórica que operacionaliza é rica e integradora. Por outro, parece-nos que o DMI se situa algures num ponto intermédio, ainda que mais próximo do pólo do auto-relato, se considerarmos o contínuo entre os métodos de auto-relato e os métodos indirectos. Uma abordagem indirecta em avaliação psicológica está em consonância com uma posição teórica que assume que a acessibilidade dos estados internos é limitada (veja-se Cogswell, 2008). Apesar da dicotomia na literatura entre métodos indirectos (como os métodos projectivos), e de auto-relatato, "é mais provável que qualquer ferramenta de avaliação se situe ao longo de um contínuo em vez de ocupar uma posição num pólo ou no outro" (Cogswell, 2008, p. 27).
Parece-nos também uma vantagem do DMI o facto de se pedir ao indivíduo para se posicionar relativamente a situações conflituais reais do diaa-dia. Muitas críticas foram feitas ao formato típico dos itens dos questionários (veja-se Kline, 1999). Independentemente da capacidade do investigador que desenvolve um questionário, um item é sempre, em parte, simplista. Seria impossível captar a riqueza da experiência humana em frases simples. Os questionários costumam interrogar acerca de situações hipotéticas "típicas" e não a cerca de situações específicas, pelo que mesmo quando quem responde deseja cooperar, a informação obtida pode ser limitada (Kline, 1999). Naturalmente que, o formato do DMI também apresenta problemas, nomeadamente o facto de ser longo e, por conseguinte, fatigar as pessoas que respondem, mas pode obviar em parte os problemas habitualmente apontados aos itens dos questionários.

No DMI são obtidos resultados para cinco escalas, avaliando cada uma delas um tipo de mecanismo de defesa. Turning Against Object (TAO) implica mecanismos que lidam com o conflito pela expressão directa e/ou indirecta de agressividade, com o objectivo de manter uma ilusão do controlo sobre as ameaças externas ou ainda de mascarar os conflitos internos que são demasiado dolorosos para serem tolerados e confrontados conscientemente (inclui mecanismos como a identificação ao agressor e o deslocamento). Projection (PRO) envolve uma atribuição de forma inconsciente por parte do indivíduo de qualidades indesejáveis do Self (traços e conteúdos negativos) ao outro, sendo que esta atribuição é utilizada para justificar a expressão de hostilidade em relação a ele. Já o tipo de defesa Principalization (PRN) envolve mecanismos que lidam com o conflito através de uma reinterpretação da realidade, recorrendo à separação do conteúdo afectivo do conteúdo ideativo ou da situação (inclui mecanismos como a intelectualização, a racionalização e o isolamento dos afectos). Turning Against Self (TAS), por sua vez, descreve a tendência do indivíduo para resolver o conflito psicológico dirigindo os comportamentos, fantasias ou sentimentos agressivos contra o Self, o que pode conduzir a compromissos defensivos auto- 
destrutivos. Por fim, Reversal (REV) avalia a tendência para responder de forma positiva ou neutra a uma situação negativa, que se esperaria desencadear uma resposta negativa por parte do indivíduo (inclui mecanismos como a negação, a formação reactiva e o recalcamento), o que resulta num empobrecimento da capacidade de percepção do perigo. Note-se que cada um dos cinco estilos ou tipos defensivos deve ser entendido como um constructo abrangente, podendo englobar uma grande variedade de respostas defensivas específicas (Ihilevich \& Gleser, 1986, 1991; Juni, 1999).

Consideramos importante referir que a perspectiva de Ihilevich e Gleser (1986), e a "arrumação" dos diferentes mecanismos de defesa em cinco grandes tipos, parece-nos conter uma limitação importante: não permite incluir em nenhum desses cinco tipos, um importante mecanismo de defesa, a clivagem, que como é sabido, consiste numa divisão e impossibilidade de integrar afectos contrários relativamente ao mesmo objecto ou ao self (McWillimas, 2004).

Em relação às características psicométricas do DMI, muitos dos estudos já realizados comprovam a sua validade enquanto instrumento psicológico capaz de avaliar mecanismos de defesa e a sua relação com a psicopatologia, a saúde, os estilos perceptivos e a intervenção terapêutica, entre outros aspectos (Andersen \& Leitner, 1991; Berman \& McCann, 1995; Cramer, 1988; Ihilevich \& Gleser, 1986, 1991; 1995; Juni, 1999). Juni (1999) concluiu que uma meta-análise relativa à validade apoia o racional teórico do teste, e Cramer (1988), numa revisão da literatura, conclui que as escalas se relacionam de forma esperada com variáveis cognitivas, de personalidade e de psicopatologia.

O DMI foi adaptado para a população portuguesa por Justo et al. (2010). Justo e colegas apresentam valores de alpha de Cronbach para as cinco escalas, e para homens e mulheres, entre .73 e .86 .

\section{O Defense Mechanisms Inventory-Youth} (DMI-Y; Ihilevich, \& Gleser, 1986) é, em tudo, estruturalmente idêntico à versão para adultos. Existem também duas versões, uma para o sexo masculino e outra para o sexo feminino, compostas igualmente por 10 vinhetas. Pode ser aplicado a sujeitos a partir dos 12 anos. O conteúdo de algumas vinhetas foi alterado de modo a adequar-se a adolescentes. Outras, foram substituídas por vinhetas que descrevem situações da vida quotidiana mais relevantes para adolescentes. Foi adaptado para a população portuguesa por Justo, Silva, Neves e Frade (2011).

Um exemplo de uma vinheta desta versão é: "Você tem que cumprir um itinerário de distribuição de jornais para muitas pessoas e tem de trabalhar muito, porque precisa de dinheiro e os empregos são raros. É da sua responsabilidade fazer com que tudo corra tranquilamente. Você tem um colega que o ajuda a fazer a entrega dos jornais. Recentemente, muitas pessoas se têm queixado de não receber os seus jornais. Você sabe que tem sido cuidadoso a fazer o seu trabalho e, por isso, decide despedir o seu ajudante. Nesse mesmo dia, o seu patrão aparece em sua casa. Sem o deixar explicar, o seu patrão diz-lhe que o itinerário de distribuição de jornais lhe é retirado porque você é descuidado. O seu ajudante fica com o seu lugar, e agora você é que é o ajudante".

"Qual seria a sua reacção (comportamento real)?"

"Aceitava de bons modos, uma vez que o meu patrão está, apenas, a cumprir as suas funções.

Censurava o meu patrão por ter tomado uma decisão contra mim, mesmo antes de se ter encontrado comigo.

Ficava agradecido por ter sido aliviado de um trabalho tão difícil.

Ficava à espera de uma oportunidade para tramar o meu ajudante.

Censurava-me por não ter sido suficientemente competente no meu trabalho."

Em Portugal, os estudos realizados com a forma para adultos do DMI foram fundamentalmente em duas áreas, gravidez e parentalidade (Justo, 1990, 1999; Justo et al., 2010) e psicopatologia, especificamente depressão (Campos, et al, 2011; Vaz, 2012) e traços disfuncionais da personalidade (Rachão \& Campos, 2015)

Foram realizados três estudos em Portugal que relacionaram as experiências depressivas anaclíticas e introjectivas e os mecanismos de defesa de acordo com a perspectiva de Ihilevich e Gleser, utilizando o DMI e o DMI-Y. Um estudo (Campos et al., 2011) foi realizado com uma amostra comunitária, outro (Vaz, 2012) utilizou 
uma amostra clínica de pacientes psiquiátricos e o terceiro (Parreira, 2013), uma amostra de adolescentes. Apesar de se registarem diferenças em função do tipo de amostra, um aspecto é significativo e comum aos resultados dos vários estudos referidos, apoiando a validade do DMI e do DMI-Y por um lado e, por outro, estando de acordo com a teoria psicanalítica. Verifica-se que as experiências depressivas propostas por Blatt se relacionam sempre com o tipo de mecanismo de defesa turning against self. A teoria psicanalítica tem vindo a referir sistematicamente o voltar $d a$ agressividade contra o próprio como $\mathrm{o}$ mecanismo psicológico central na personalidade depressiva (e.g., Coimbra de Matos, 2001, Freud, 1917; Goldstein \& Anthony, 1988), o que protege o sujeito de consciencializar a malevolência do objecto. Freud (1917) havia já descrito de forma brilhante este núcleo central de cariz masoquista da personalidade depressiva em Luto $e$ Melancolia, ao referir-se à introjecção da agressividade e à identificação do Ego ao objecto na sequência da perda $\mathrm{e}$, ao facto da raiva contra $\mathrm{o}$ objecto abandonante refluir sobre o próprio.

\section{O Children Aperception Test na avaliação dos mecanismos de defesa em crianças}

A avaliação de mecanismos de defesa através de métodos projectivos, como o Children Aperception Test (CAT, Bellak \& Bellak, 1949, 1950), afigura-se importante porque, instrumentos como o DMI ou DSQ não permitem a avaliação de crianças. O CAT é uma técnica projectiva temática, aplicada normalmente a crianças entre os 3 e os 10 anos. Existem duas formas, o CAT figuras animais e o CAT figuras humanas. São ambas compostas por 10 cartões. As situações representadas são as mesmas, diferindo apenas no facto dos personagens serem animais ou figuras humanas. A tarefa da criança consiste em contar histórias sobre as cenas representadas. Pela análise destas histórias pode ver-se como a criança lida com determinadas problemáticas, conflitos ou temáticas universais no desenvolvimento infantil, como por exemplo a rivalidade fraterna e a agressividade, bem como quais os mecanismos de defesa que utiliza preferencialmente. Note-se que a versão com figuras humanas do CAT (Bellak \& Hurvich, 1970; Bellak, 1975) foi estudada em Portugal nos anos 80 por Silva (1982) que trabalhou com o "sistema" de análise aperceptiva de Rosenzweig e Fleming (1949) e de análise temática de Eron (1965). A observação dos dados normativos obtidos por Silva (1982) pode ser útil na medida em que faculta ao intérprete referências sobre o desempenho típico das crianças face aos cartões, independentemente de outros instrumentos de análise poderem ser usados.

Cramer (1991) propôs uma grelha de análise, o Defense Mechanism Manual, para o Tematic Aperception Test (TAT) e para o CAT que permite codificar mecanismos de defesa utilizando as respostas/histórias dadas a alguns dos cartões. No caso do CAT sugere que se utilizem as respostas aos cartões 3,5 e 10. As defesas a codificar estão naturalmente em acordo com o seu modelo teórico anteriormente mencionado e são: a negação, a projecção e a identificação. O sistema apresenta alguma complexidade e exige treino inicial. Prevê que para cada uma das três categorias de defesas deva ser considerada a eventual presença nas histórias de sete diferentes processos psicológicos. São exemplos para a Negação, a Omissão e a Negação da realidade, para a projecção, o Pensamento mágico ou circunstancial e a História ou tema muito inusual ou bizarro, e para a Identificação, o Moralismo e o Adiamento da Gratificação.

A grelha de Boekholt (2000) da escola psicanalítica francesa assenta também numa visão maturacional ou evolutiva dos mecanismos de defesa, mecanismos estes que permitiriam, ao ser avaliados, esboçar uma proposta estruturalevolutiva de diagnóstico em três níveis: organização psicótica, organização desarmónica e organização neurótica. A lógica subjacente é de uma análise mais estrutural das respostas, e não, como os autores da prova enfatizaram, uma análise de conteúdo do CAT - figuras animais (Bellak \& Bellak, 1949). Assim sendo, o discurso e os comportamentos da criança na situação de teste CAT, podem ser decompostos em procedimentos, ou seja, o discurso e o comportamento são traduzidos, o mesmo é dizer decompostos, em segmentos de análise, que podem ser classificados mediante a correspondência com procedimentos de elaboração das narrativas, de acordo com uma grelha de análise. 
São sete os tipos de procedimentos utilizados de acordo com Boekholt (2000, p. 192):

Os procedimentos que traduzem o recurso à esfera motora e corporal.

Exemplos:

Procedimento MC3, Risos, mímicas, caretas e onomatopeias;

Procedimento MC4, Participação corporal, deslocações, gestos.

Os procedimentos que traduzem o recurso à relação com o clínico.

Exemplos:

Procedimentos $\mathrm{RC} 1$, Procura de proximidade corporal;

Procedimentos RC4, Auto-depreciação, autovalorização.

Os procedimentos que traduzem o recurso à realidade exterior.

Exemplos:

Procedimento RE1, Recurso à evidência, apego ao conteúdo manifesto;

Procedimento RE4, Insistência nas qualidades sensoriais do material.

Os procedimentos que traduzem o recurso ao evitamento e à inibição.

Exemplos:

Procedimento EI1, Restrição, silêncios, recusa, tendenciais para recusa, necessidade de fazer perguntas;

Procedimento EI2, Anonimato, razões dos conflitos não esclarecidas, colagens, banalização.

Os procedimentos que traduzem o recurso ao afecto.

Exemplos:

Procedimento RA1, Expressão verbalizada de afectos

Procedimento RA4, Afectos inadequados, afectos maciços.

Os procedimentos que traduzem o recurso ao imaginário e à fantasia.

Exemplos:

Procedimento IF1, Introdução de personagens que não figuram na imagem;

Procedimento IF2, Apelo ao imaginário infantil dos contos.

Os procedimentos que traduzem o recurso à objectividade e ao controlo.

Exemplos:

Procedimento OC1, Descrição com apego aos pormenores;
Procedimento OC4, Anulação.

Com base no tipo de procedimentos utilizados é possível avaliar as defesas em jogo. Boekholt (2000) descreve as defesas primitivas: projecção, clivagem e negação; as defesas ligadas à elaboração da posição depressiva: recusa maníaca e defesas narcísicas; as defesas neuróticas: de tipo fóbico, ligadas ao recalcamento e as defesas rígidas. Esta última divisão tripartida, é compatível com o modelo triplo da neurose: fóbica, histérica e obsessiva. A contabilização do tipo de defesas poderá, eventualmente, permitir (ou não) uma proposta de diagnóstico estrutural. É claro que a atribuição de um diagnóstico tendo por base esta contabilização poderá implicar um salto inferencial importante e, em última instância, apresentar problemas do ponto de vista da validade, muito em particular se não for realizada com um forte apoio dos dados provenientes de outras fontes, como a observação, a entrevista e a anamnese. A complexidade e a subjectividade da análise das narrativas e da utilização da grelha de procedimentos é também, e só por si, uma limitação desta abordagem de avaliação dos mecanismos defesa e faz notar a exigência de formação prévia, no sentido de minimizar eventuais problemas de precisão inter-cotadores. A riqueza da informação obtida a cerca dos mecanismos defensivos que são privilegiados pela criança a quem é aplicado o CAT, pode, no entanto, relativizar as dificuldades deste sistema de codificação desta prova temática.

\section{Conclusão}

Alguns clínicos adoptam uma abordagem psicodinâmica na avaliação do funcionamento psíquico e do mal-estar psicológico (Campos, 2017). Esta abordagem permite uma compreensão rica e integradora e, fornece um contexto teórico para a leitura das queixas dos indivíduos avaliados. Nesta lógica, a avaliação dos mecanismos de defesa, para além da avaliação de outros elementos do funcionamento psíquico, como as representações objectais e do self, assume um papel fundamental (e. g., Campos, 2017). Naturalmente que muitas vezes, os clínicos avaliam os mecanismos de defesa de forma mais "informal", através do material obtido em entrevista. A utilização ou não de provas 
psicológicas numa determinada avaliação depende, na verdade, de vários aspectos (veja-se Pallás \& Lorens, 2010), nomeadamente de opções teóricas e de aspectos pragmáticos, como o tempo disponível, mas pode ter claras vantagens, como a objectividade e a possibilidade de comparação com dados normativos. Provas de resposta fechada ou, por outro lado, de escolha não forçada, podem ser utilizadas na avaliação dos mecanismos de defesa, como são o caso do DMI e do CAT, respectivamente.

Destaca-se como vantagens do DMI a formulação teórica subjacente, integradora e bem fundamentada, e o facto de se pedir aos indivíduos para se posicionarem relativamente a situações conflituais reais do dia-a-dia, e não face a itens com o formato habitual de questionário. No entanto, a extensão do instrumento pode conduzir à desmotivação e à falta de cooperação das pessoas que respondem. Por outro lado, pode argumentar-se que a formulação teórica que sustenta a prova exclui um importante mecanismo de defesa, a clivagem.

Relativamente ao sistema de codificação de Boekholt (2000) para o CAT, pode dizer-se que a complexidade, subjectividade e exigência de treino prévio tornam a sua utilização na prática clínica uma tarefa exigente. Ainda assim, permite obter informação sobre mecanismos de defesa na criança. $\mathrm{Na}$ ausência de provas de resposta fechada para avaliar este constructo nesta faixa etária, poderá ser uma boa opção para o clínico. A situação CAT é geralmente bem aceite pelas crianças e as histórias contadas podem ser utilizadas, não apenas para avaliar mecanismos de defesa, mas também para avaliar outros aspectos do funcionamento psíquico com relevância neste período de desenvolvimento.

\section{Referências}

Andersen, T., \& Leitner, J. M. (1991). The relationship between the Defense Mechanisms Inventory and reported symptomatology in college females. Personality and Individual Differences, 12, 967-969.

Andrews, G., Singh M., \& Bond, M. (1993). The Defense Style Questionnaire. Journal of Nervous and Mental Disease, 181, 246-256.
Azibo, A. (2007). Mechanisms of defense: Nepenthe theory and psychiatric symptomatology. The Negro Educational Review, 58, 49-69.

Bellak, L. (1975). The TAT, CAT and SAT in clinical use. New York: Grune \& Stratton.

Bellak L., \& Bellak, S. (1949). The Children Aperception Test. New York: C.P.S. Company.

Bellak, L., \& Bellak, S. S. (1950). An introductory note on the Children's Apperception Test (CAT). Journal of Projective Techniques, 14, 173-180.

Bellak, L., \& Hurvich, M. S. (1970). Children's Aperception Test (Human Figures): Manual. New York: C.P.C., Inc.

Berman, S. M. W., \& McCann, J. T. (1995). Defense mechanisms and personality disorders: An empirical test of Millon's theory. Journal of Personality Assessment, 64, 132-144.

Boekholt, M. (2000). Provas temáticas na clínica infantil. Lisboa: Climepsi.

Bond, M., Gardner, S. T., Christian, J., \& Sigal, J. J. (1983). Empirical study of self-rated defense styles. Archives of General Psychiatry, 40, 333-338.

Bowins, B. (2006). How psychiatric treatments can enhance defense mechanisms. American Journal of Psychiatry, 66, 173-194.

Bowins, B. (2010). Personality disorders: A dimensional defense mechanism approach. American Journal of Psychotherapy , 64, 153169.

Campos, R. C. (2017). Do processo de avaliação da personalidade em contextos clínicos ao diagnóstico psicodinâmico: Contributos para uma avaliação psicológica psicodinâmica. Revista Iberoamericana de Diagnóstico y Evaluación - e Avaliação Psicológica, 4, 4456

Campos, R. C., Besser, A., \& Blatt, S. J. (2011). The Relationships between defenses and experiences of depression: An exploratory study. Psychoanalytic Psychology, 28, 196208.

Chabrol, H., \& Leichsenring, F. (2006). Borderline personality organization and psychopathic traits in nonclinical adolescents: Relationship of identity diffusion, primitive 
defense mechanisms and reality testing with callousness and impulsivity traits. Bulletin of the Menninger Clinic, 70, 160-170.

Chávez-León, E., Muñoz, M., \& Uribe, M. (2006). An empirical study of defense mechanisms in panic disorder. Salud Mental, 29, 15-22.

Cogswell, A. (2008). Explicit rejection of an implicit dichotomy: Integrating two approaches to assessing dependency Journal of Personality Assessment, 90, 26-35.

Coimbra de Matos, A. (2001). Depressão: Episódios de um percurso em busca do seu sentido. Lisboa: Climepsi.

Cooper, S. H., Perry, J. C., \& Arrow, D. (1988). An empirical approach to the study of defense mechanisms: I. Reliability and preliminary validity of Rorschach Defense Scales. Journal of Personality Assessment, 52, 187-203.

Cramer, P. (1988). The Defense Mechanisms Inventory: A review of research and discussion of the scales. Journal of Personality Assessment, 52, 142-154.

Cramer, P. (1991). The development of defense mechanisms: Theory, research, and assessment. New York: Springer-Verlag.

Cramer, P. (1998). Coping and defense mechanisms: What's the difference? Journal of Personality, 66, 919-946.

Cramer, P. (1999a). Personality, personality disorders, and defense mechanisms. Journal of Personality, 67, 535-554.

Cramer, P. (1999b). Change in defense mechanisms during psychoanalysis and psychotherapy: A case study. In Colhler, B., \& Cohen, J. (Eds), The psychoanalytic study of lives over time: Clinical and research perspective on children who return to treatment in adulthood (pp. 309-330). Academic Press.

Cramer, P. (2000). Defense mechanisms in Psychology today. The American Psychologist, 55, 637-646.

Cramer, P. (2002). Defense mechanisms, behavior, and affect in young adulthood. Journal of Personality, 70, 103-126.

Cramer, P. (2005). Another "lens" for understanding therapeutic change: The interaction of IQ with defense mechanisms. In J. S. Auerbach, K. N. Levy, \& C. E. Schaffer
(Eds.) Relatedness, self-definition and mental representation: Essays in honour of Sidney J. Blatt (pp. 120-133). New York: Routledge.

Cramer, P. (2006). Protecting the Self: Defense mechanisms in Action. New York: Guilford Press.

Cramer, P., \& Blatt, S. J. (1990). Use of TAT to measure changes in defense mechanisms following intensive psychotherapy. Journal of Personality Assessment, 54, 236-251.

Cramer, P., \& Blatt, S. J. (1993). Change in defense mechanisms following intensive treatment, as related to personality organization and gender. In W. Ehlers, U. Hentschel, G. Smith, \& J. G. Draguns (Eds.), The concept of defense mechanisms in contemporary Psychology (pp. 310-320). New York: Springer-Verlag.

D'Angelo, A., San Martini, P., \& Tomasello, M. A. (2000). Psychometric characteristics of a Likert version of the Defense Mechanisms Inventory. Bollettino di Psicologia Applicata, 230, 47-56.

Drageset, S., \& Lindstrom, T. C. (2003). The mental health of women with suspected breast cancer: The relationship between social support, anxiety, coping and defences in maintaining mental health. Journal of Psychiatry and Mental Health Nursing, 10, 401-409.

Flett, G. L., Besser A., \& Hewitt, P. L. (2005). Perfectionism, ego defense style and depression: A comparison of self-reports versus informant ratings. Journal of Personality, 73, 1355-1396.

Freud, S. (1917/1980). Mourning and Melancholia (Edição standard brasileira das obras psicológicas completas de Sigmund Freud - vol. XIV). Rio de Janeiro: Imago Editora.

Freud, S. (1926/1980). Inibições, sintomas $e$ angústia (Edição standard brasileira das obras psicológicas completas de Sigmund Freud vol. XX). Rio de Janeiro: Imago Editora.

Freud, A. (1946). The ego and the mechanisms of defense. New York: International Universities Press.

Freud, A. (1965). Normality and pathology in childhood. New York: International Universities Press. 
Gleser, G. C., \& Ihilevich, D. (1969). An objective instrument for measuring defense mechanisms. Journal of Consulting and Clinical Psychology, 33, 51-60.

Goldstein, W. N., \& Anthony, R. N. (1988). The diagnosis of depression and the DSMs. American Journal of Psychotherapy, 42, 180196.

Gould, J. R., Prentice, N. M., \& Ainslie, R. C. (1996). The Splitting Index: Construction of a scale measuring the defense mechanism of splitting. Journal of Personality Assessment, 66, 414-430.

Haan, N. (1963). Proposed model of ego functioning: Coping and defense mechanisms in relationship to I.Q. change. Psychological Monographs, 77, 1-23.

Hovanesian, S., Isakov, I., \& Cervellione, K. L. (2009). Defense mechanisms and suicide risk in major depression. Archives of Suicide Research, 13, 74-86.

Ihilevich, D., \& Gleser, G. C. (1986). Defense mechanisms: Their classification, correlates, and measurement with the Defense Mechanisms Inventory. Owosso: DMI Associates.

Ihilevich, D., \& Gleser, G. C. (1991). Defenses in psychotherapy: The clinical application of the Defense Mechanisms Inventory. Owosso: DMI Associates.

Ihilevich, D., \& Gleser, G. C. (1995). The Defense Mechanisms Inventory: Its developmental and clinical applications. In Conte, H., \& Plutchik, R. (Eds), Ego Defenses: Theory and measurement (pp 221246). New York: Einstein Psychiatry Publication.

Juni, S. (1999). The Defense Mechanisms Inventory: Theoretical and psychometric implications. Current Psychology, 17, 313332.

Justo, J. M. (1990). Gravidez e mecanismos de defesa: Um estudo introdutório. Análise Psicológica, 8, 371-376.

Justo, J. (1999). Evolução psicológica ao longo da gravidez e puerpério: Um estudo transversal. Revista Portuguesa de Psicossomática, 1, 115-129.

Justo, J. M., Melo, V., \& Ferreira, A. S. (2010). Defence mechanisms of infertile couples vs. fertile couples. International Journal of Developmental and Educational Psychology, 2, 433 - 442.

Justo, J. M., Silva, A. L., Neves, A. L., \& Frade, C. L. (2011). The Portuguese version of the adolescents form of the Defense Mechanisms Inventory. International Journal of Developmental and Educational Psychology, 3, 121-134.

Kernberg, O. (1985). Borderline conditions and pathological narcissism. Oxford: Jason Aronson.

Kline, P. (1999). Handbook of psychological testing. London: Routledge.

Kragh, U. (1955). The Actual-Genetic Model of Perception-Personality. Lund: Gleerup

Kreitler, H., \& Kreitler, S. (1972). Cognitive determinants of defensive behavior. British Journal of Social and Clinical Psychology, 11, 359-372.

Kwon, P., \& Lemon, K. E. (2000). Attributional style and defense mechanisms: A synthesis of cognitive and psychodynamic factors in depression. Journal of Clinical Psychology, 56, 723-735.

La Gruta, S., et al. (2006). Predictive signs and indicators of aggressiveness and violence: $\mathrm{A}$ comparison between a group of adolescents attending an external penal area, a group of prisoners and a group of patients with borderline personality disorder. Minerva Pediatrica, 58, 121-129.

Laplanche, J., \& Pontalis, J. B. (1988). The Language of Psychoanalysis. London: Karnac.

Macmillan, N. A., \& Creelman, C. D. (2005). Detection theory: A user's guide (2nd ed.). Mahwah, NJ, US: Lawrence Erlbaum Associates Publishers.

McWilliams, N. (2004). Formulação psicanalítica de casos. Lisboa: Climepsi.

Moreira, J. M. (2004). Questionários: Teoria e prática. Coimbra: Almedina,

Mullen, L., Blanco, C., Vaughan, S., Vaughan, S., \& Roose, S. (1999) Defense mechanisms and personality in depression. Depression and Anxiety, 10, 168-174.

Oliveira, A. M. (2012). As relações de objecto/estilos de vinculação e os mecanismos de defesa na personalidade depressiva. Tese 
de Doutoramento em Psicologia Clínica, Universidade de Évora.

Pallás, C. M., \& Lorens, O. G. (2010). Enfoque de la evaluación psicológica de la Revista Iberoamericana de Diagnóstico y Evaluación Psicológica (Ridep). Revista Iberoameircana de Diagnóstico e Evaluacion - e Avaliação Psicológica, 30, 35-55.

Parreira, T. I. (2013). Mecanismos de defesa e experiências depressivas: Um estudo com uma amostra de adolescentes. Dissertação de Mestrado em Psicologia Clínica e da Saúde, Universidade de Évora.

Perry, L. C., \& Cooper, S. H. (1986). A preliminary report on defense and conflicts associated with borderline personality disorder. Journal of the American Psychoanalytic Association, 34, 863-893.

Perry, J. C., \& Kardos, M. (1995). A review of the Defense Mechanism Rating Scales. In H. R. Conte, \& R. Plutchik (Eds.), Ego defenses: Theory and measurement (pp. 283-299). New York: Wiley-Interscience.

Rachão, I., \& Campos, R. C. (2015). Personality styles and defense mechanisms in a community sample of adolescents: An exploratory study. Bulletin of the Menninger Clinic, 79, 14-40.

Ruuttu, T. et al. (2006). Psychometric properties of the defense style questionnaire (DSQ-40) in adolescents. Journal of Nervous and Mental Diseases, 194, 98-105.

Schauenburg, H., Willenborg, V., Sammet, I., \& Ehrenthal, J. (2007). Self-reported defence mechanisms as an outcome measure in psychotherapy: A study on the German version of the Defense Style Questionnaire DSQ 40. Psychology and Psychotherapy: Theory, Research and Practice, 80, 355-366.

Segal, D., Coolidge, F., \& Mizuno, H. (2007). Defense mechanism differences between younger and older adults: A cross-sectional investigation. Aging \& Mental Health, 11, 415-422.

Silva, D. R. (1982). O Teste de apercepção para Crianças (Figuras Humanas) CAT-H: Um estudo de normas. Lisboa: Instituto de Investigação Científica.

Silva, A. S. M. (2014). Patologia borderline: Representações relacionais vulnerabilidades do Self. Tese de doutoramento em Psicologia Clínica, Faculdade de Psicologia, Universidade de Lisboa.

Thygesen, K. L., Drapeau, M., Trijsburg, W., Lecours, S., \& Roten, Y. (2008). Assessing defense styles: Factor structure and psychometric properties of the New Defense Style Questionnaire 60 (DSQ-60). International Journal of Psychology and Psychological Therapy, 8, 171-181.

Trijsburg R. W., Bond, M., \& Drapeau, M. (2003) Defense Style Questionnaire-60: Unpublished instrument. Institute of Community and Family Psychiatry, McGill University.

Vaillant, G. E. (1971). Theoretical hierarchy of adaptive Ego mechanisms: A 30 year followup of men selected for psychological health. Archives of General Psychiatry, 24, 107-118.

Vaillant, G. E. (1977). Adaptation to life. Boston: Little Brown.

Vaillant, G. E. (1992) Ego Mechanisms of defense: A guide for clinicians and researchers. Washington, DC: American Psychiatric Press, Inc.

Vaillant, G. E., \& Drake, R. R. (1985). Maturity of Ego defences in relation to DSM-III Axis II personality disorder. Archives of General Psychiatry, 42, 597-601.

Vaz, S. F. C. F. (2012). Mecanismos de defesa e experiências depressivas: Um estudo com uma amostra clínica. Dissertação de Mestrado em Psicologia Clínica e da Saúde, Universidade de Évora. 